\title{
SOME METRIC PROPERTIES OF SPACES OF STABILITY CONDITIONS
}

\author{
JON WOOLF
}

\section{INTRODUCTION}

The space of locally-finite stability conditions $\operatorname{Stab}(\mathcal{C})$ on a triangulated category $\mathcal{C}$ is a (possibly inifinite-dimensional) complex manifold. There is a metric which induces the topology. For numerically-finite $\mathcal{C}$, for instance the coherent bounded derived category of a smooth complex projective variety or the bounded derived category of a finite-dimensional algebra, one can consider the finite-dimensional submanifold of numerical stability conditions. We show that under mild conditions this is complete in the metric and describe limiting stability conditions.

The contents are as follows: $₫ 2$ contains some background material on stability conditions. Section 3 contains the proof that a full component of the space of stability conditions whose central charges factor through some finite rank quotient $A$ of the Grothendieck group is complete. Recall that full means that the space of stability conditions is locally homeomorphic to $\operatorname{Hom}(A, \mathbb{C})$, and not to some proper subspace. The key result is Proposition 3.6 which describes the limiting Harder-Narasimhan filtrations. The assumptions are satisified, for instance, for a full component of the space of numerical stability conditions on a numerically-finite triangulated category.

In $\$ 4$ we independently verify that the metric on the space of numerical stability conditions on a smooth complex projective curve of genus $\geq 1$ is complete. We compute this metric as follows. There is a natural action of the universal cover $G$ of $G L_{2}^{+} \mathbb{R}$ on any space of stability conditions. When the phases of semistable objects are dense for a stability condition $\sigma$, the orbit through $\sigma$ is free and the restriction $d_{G}$ of the metric to it is independent of $\sigma$ and can be explicitly described, and seen to be complete. In the case of curves of genus $g \geq 1$ this density of phases condition is satisfied and the action of $G$ is both free and transitive, so that the space of numerical stability conditions is isometric to $\left(G, d_{G}\right)$. The metric $d_{G}$ is closely related to the hyperbolic metric on the upper half plane - the universal cover of the conformal linear maps forms a subgroup of $G$ isomorphic to $\mathbb{C}$ and the quotient can be identified with the upper half-plane in such a way that the quotient metric is half the standard hyperbolic metric — see Proposition 4.1.

Section 5 contains some observations about relationships between the hearts of stability conditions. Corollary 5.2 states that hearts of stability conditions in the same component of $\operatorname{Stab}(\mathcal{C})$ are related by finite sequences of tilts. In the process of proving Theorem 3.7 we obtain a description of the limiting stability condition

Date: August 2011.

I would like to thank the Newton Institute, Cambridge, for their generous hospitality in April and May 2011, when this paper was completed. I would also like to thank Tom Bridgeland and Arend Bayer for helpful conversations. 
$\sigma$ of a convergent sequence $\sigma_{n}$. As a consequence we obtain Corollary 5.3, which states that if the $\sigma_{n}$ all have the same heart, $\mathcal{A}$ say, then the heart of $\sigma$ must be a right tilt of $\mathcal{A}$.

\section{Stability CONDITIONS}

We fix some notation. Let $\mathcal{C}$ be an additive category. We write $c \in \mathcal{C}$ to mean $c$ is an object of $\mathcal{C}$. We will use the term subcategory to mean strict, full subcategory. When $\mathcal{C}$ is triangulated with shift functor [1] exact triangles will be denoted either by $a \rightarrow b \rightarrow c \rightarrow a[1]$ or by a diagram

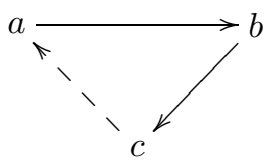

where the dotted arrow denotes a morphism $c \rightarrow a[1]$. We will always assume that $\mathcal{C}$ is essentially small so that isomorphism classes of objects form a set. Given sets $S_{i}$ of objects for $i \in I$ let $\left\langle S_{i} \mid i \in I\right\rangle$ denote the ext-closed subcategory generated by objects isomorphic to an element in some $S_{i}$. We use the same notation when the $S_{i}$ are subcategories of $\mathcal{C}$.

Let $\mathcal{C}$ be a triangulated category and $K(\mathcal{C})$ be its Grothendieck group. A stability condition $\sigma=\left(\mathcal{Z}_{\sigma}, \mathcal{P}_{\sigma}\right)$ on $\mathcal{C}$ [2, Definition 1.1] consists of an additive group homomorphism $\mathcal{Z}_{\sigma}: K(\mathcal{C}) \rightarrow \mathbb{C}$ and full additive subcategories $\mathcal{P}_{\sigma}(\varphi)$ of $\mathcal{C}$ for each $\varphi \in \mathbb{R}$ satisfying

(1) if $c \in \mathcal{P}_{\sigma}(\varphi)$ then $\mathcal{Z}_{\sigma}(c)=m(c) \exp (i \pi \varphi)$ where $m(c) \in \mathbb{R}_{>0}$;

(2) $\mathcal{P}_{\sigma}(\varphi+1)=\mathcal{P}_{\sigma}(\varphi)[1]$ for each $\varphi \in \mathbb{R}$;

(3) if $c \in \mathcal{P}_{\sigma}(\varphi)$ and $c^{\prime} \in \mathcal{P}_{\sigma}\left(\varphi^{\prime}\right)$ with $\varphi>\varphi^{\prime}$ then $\operatorname{Hom}\left(c, c^{\prime}\right)=0$;

(4) for each nonzero object $c \in \mathcal{C}$ there is a Harder-Narasimhan filtration, i.e. a finite collection of triangles

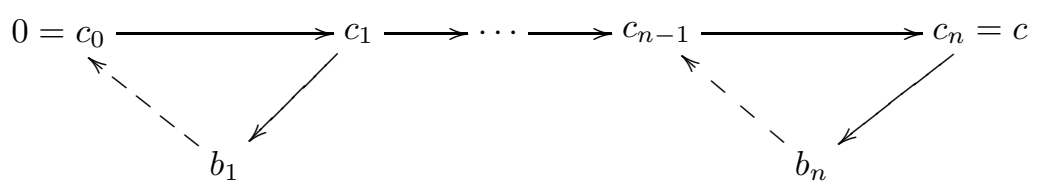

with $b_{j} \in \mathcal{P}_{\sigma}\left(\varphi_{j}\right)$ where $\varphi_{1}>\cdots>\varphi_{n}$.

The homomorphism $\mathcal{Z}_{\sigma}$ is known as the central charge and the objects of $\mathcal{P}_{\sigma}(\varphi)$ are said to be $\sigma$-semistable of phase $\varphi$. The objects $b_{j}$ are known as the $\sigma$-semistable factors of $c$. We define $\varphi_{\sigma}^{+}(c)=\varphi_{1}$ and $\varphi_{\sigma}^{-}(c)=\varphi_{n}$. The mass of $c$ is defined to be $m_{\sigma}(c)=\sum_{i=1}^{n} m\left(b_{i}\right)$.

Lemma 2.1. If $a \rightarrow b \rightarrow c \rightarrow a[1]$ is an exact triangle then

$$
\min \left\{\varphi_{\sigma}^{-}(a), \varphi_{\sigma}^{-}(c)\right\} \leq \varphi_{\sigma}^{-}(b) \leq \varphi_{\sigma}^{+}(b) \leq \max \left\{\varphi_{\sigma}^{+}(a), \varphi_{\sigma}^{+}(c)\right\}
$$

for any stability condition $\sigma$.

Proof. Note that $\varphi_{\sigma}^{+}(b)=\sup \left\{t \mid \exists b^{\prime} \in \mathcal{P}_{\sigma}(t)\right.$ with $\left.\operatorname{Hom}\left(b^{\prime}, b\right) \neq 0\right\}$ and similarly

$$
\varphi_{\sigma}^{-}(b)=\inf \left\{t \mid \exists b^{\prime} \in \mathcal{P}_{\sigma}(t) \text { with } \operatorname{Hom}\left(b, b^{\prime}\right) \neq 0\right\} \text {. }
$$

Furthermore, both the extreme values are achieved. Suppose $\varphi_{\sigma}^{+}(b)>\varphi_{\sigma}^{+}(c)$. There is a semistable $b^{\prime}$ with phase $\varphi_{\sigma}^{+}(b)$ and nonzero morphism $b^{\prime} \rightarrow b$. Since 
$\operatorname{Hom}\left(b^{\prime}, c\right)=0$ for phase reasons this morphism factors through a nonzero morphism to $a$. Hence $\varphi_{\sigma}^{+}(a) \geq \varphi_{\sigma}^{+}(b)$ and $\varphi_{\sigma}^{+}(b) \leq \max \left\{\varphi_{\sigma}^{+}(a), \varphi_{\sigma}^{+}(c)\right\}$. The other inequality is proved similarly.

By a $\sigma$-filtration of $0 \neq c \in \mathcal{C}$ we mean a filtration as above whose factors are $\sigma$ semistable. The Harder-Narasimhan filtration is one example; in general there will be others not satisfying the decreasing phase of factors condition (which uniquely characterises the Harder-Narasimhan filtration). One consequence of the previous lemma is that the Harder-Narasimhan filtration has minimal phase range amongst all $\sigma$-filtrations in the sense that if a $\sigma$-filtration of $c$ has factors with phases $\varphi_{i}$ then

$$
\min \left\{\varphi_{i}\right\} \leq \varphi_{\sigma}^{-}(c) \leq \varphi_{\sigma}^{+}(c) \leq \max \left\{\varphi_{i}\right\}
$$

For any interval $I \subset \mathbb{R}$ let $\mathcal{P}_{\sigma}(I)=\left\langle\mathcal{P}_{\sigma}(\varphi) \mid \varphi \in I\right\rangle$ be the full subcategory generated by $\sigma$-semistable objects with phases in $I$. For any $t \in \mathbb{R}$ the full subcategory $\mathcal{P}_{\sigma}(t, \infty)$ is a $t$-structure. In particular the heart

$$
\mathcal{P}_{\sigma}(t, \infty) \cap \mathcal{P}_{\sigma}(t, \infty)^{\perp}[1]=\mathcal{P}_{\sigma}(t, \infty) \cap \mathcal{P}_{\sigma}(-\infty, t+1]=\mathcal{P}_{\sigma}(t, t+1]
$$

is an abelian subcategory of $\mathcal{C}$. Note that $Z_{\sigma}(a) \neq 0$ for any $0 \neq a \in \mathcal{P}_{\sigma}(t, t+1]$ so that the phase

$$
\varphi_{\sigma}(a)=\frac{1}{\pi} \arg Z_{\sigma}(a) \in(t, t+1]
$$

is well-defined. The semistable objects in $\mathcal{P}_{\sigma}(t, t+1]$ can be characterised as those $b$ such that $a \longmapsto b$ implies $\varphi_{\sigma}(a) \leq \varphi_{\sigma}(b)$, or equivalently those for which $b \rightarrow c$ implies $\varphi_{\sigma}(b) \leq \varphi_{\sigma}(c)$. See [2, §5] for details. The abelian category $\mathcal{P}_{\sigma}(0,1]$ is referred to as the heart of the stability condition $\sigma$.

When the length $|I|=\sup I-\inf I$ of the interval is $<1$ the category $\mathcal{P}_{\sigma}(I)$ is quasi-abelian, see [2, Lemma 4.3]. The strict short exact sequences arise from exact triangles $a \rightarrow b \rightarrow c \rightarrow a[1]$ in $\mathcal{C}$ for which $a, b$ and $c$ are in $\mathcal{P}_{\sigma}(I)$. A stability condition $\sigma$ is said to be locally-finite if given $t \in \mathbb{R}$ we can find $\epsilon>0$ such that the quasi-abelian category $\mathcal{P}_{\sigma}(t-\epsilon, t+\epsilon)$ is both artinian and noetherian. (Such a category is called finite length in [2] but we prefer to avoid this terminology to avoid confusion with the a priori stronger notion of length category introduced in the next section.)

The set of locally-finite stability conditions can be topologised so that it is a, possibly infinite-dimensional, complex manifold $\operatorname{Stab}(\mathcal{C})$ referred to as the space of stability conditions on $\mathcal{C}$. For each component of $\operatorname{Stab}(\mathcal{C})$ there is a linear subspace $V \subset \operatorname{Hom}(K(\mathcal{C}), \mathbb{C})$ such that the projection $(\mathcal{Z}, \mathcal{P}) \mapsto \mathcal{Z}$ is a local homeomorphism from that component to $V$ [2, Theorem 1.2].

The topology on $\operatorname{Stab}(\mathcal{C})$ arises from the generalised metric

$$
d(\sigma, \tau)=\sup _{0 \neq c \in \mathcal{C}} \max \left\{\left|\varphi_{\sigma}^{-}(c)-\varphi_{\tau}^{-}(c)\right|,\left|\varphi_{\sigma}^{+}(c)-\varphi_{\tau}^{+}(c)\right|,\left|\log \frac{m_{\sigma}(c)}{m_{\tau}(c)}\right|\right\}
$$

which takes values in $[0, \infty]$, see [2, Proposition 8.1]. Note that $\{\tau \mid d(\sigma, \tau)<\infty\}$ is both open and closed and so is a union of components. Hence $d(\sigma, \tau)=\infty$ implies that $\sigma$ and $\tau$ are in distinct components of $\operatorname{Stab}(\mathcal{C})$. For the rest of this paper we will loosely refer to $d$ as a metric.

The group $\operatorname{Aut}(\mathcal{C})$ of automorphisms of $\mathcal{C}$ acts on the left of $\operatorname{Stab}(\mathcal{C})$ by isometries via

$$
(\mathcal{Z}, \mathcal{P}) \mapsto\left(\mathcal{Z} \circ \alpha^{-1}, \alpha \circ \mathcal{P}\right)
$$


There is also a smooth right action of the universal cover $G$ of $G L_{2}^{+} \mathbb{R}$. An element $g \in G$ corresponds to a pair $\left(T_{g}, \theta_{g}\right)$ where $T_{g}$ is the projection of $g$ to $G L_{2}^{+} \mathbb{R}$ under the covering map and $\theta_{g}: \mathbb{R} \rightarrow \mathbb{R}$ is an increasing map with $\theta_{g}(t+1)=\theta_{g}(t)+1$ which induces the same map as $T_{g}$ on the circle $\mathbb{R} / 2 \mathbb{Z}=\mathbb{R}^{2}-\{0\} / \mathbb{R}_{>0}$. In these terms the action is given by

$$
(\mathcal{Z}, \mathcal{P}) \mapsto\left(T_{g}^{-1} \circ \mathcal{Z}, \mathcal{P} \circ \theta_{g}\right)
$$

(Here we think of the central charge as valued in $\mathbb{R}^{2}$.) This action preserves the semistable objects, and also preserves the Harder-Narasimhan filtrations of all objects. The subgroup consisting of pairs for which $T$ is conformal is isomorphic to $\mathbb{C}$ with $\lambda \in \mathbb{C}$ acting via

$$
(\mathcal{Z}, \mathcal{P}) \mapsto(\exp (-i \pi \lambda) \mathcal{Z}, \mathcal{P}(\varphi+\operatorname{re} \lambda))
$$

i.e. by rotating the phases and rescaling the masses of semistable objects. This action is free and preserves the metric. The action of $1 \in \mathbb{C}$ corresponds to the action of the shift automorphism [1].

The restriction of the metric to an orbit of this $\mathbb{C}$ action is given by

$$
d(\sigma, \sigma \lambda)=\max \{|\operatorname{re} \lambda|, \pi|\operatorname{im} \lambda|\} .
$$

No sphere in the metric intersects the orbit in a smooth submanifold. Therefore $d$ is not the path metric of a Riemannian metric. Straight lines in $\mathbb{C}$ are geodesics since they have length exactly the distance between their endpoints. By considering geodesic triangles in an orbit we see that $\operatorname{Stab}(\mathcal{C})$ can never have strictly negative curvature, although it may have non-positive curvature.

Another elementary observation is that the orbits of the $\mathbb{C}$ action are closed. Hence there is an induced quotient metric $\bar{d}$ on $\operatorname{Stab}(\mathcal{C}) / \mathbb{C}$ defined by

$$
\bar{d}(x \mathbb{C}, y \mathbb{C})=\inf \{d(x, y \lambda) \mid \lambda \in \mathbb{C}\} .
$$

In $\$ 4$ we will see examples in which this quotient metric arises from a Riemannian metric with constant strictly negative curvature. It would be interesting to see whether the quotient metric has similar good properties in other examples.

\section{Completeness of SpaCes of Stability CONDitions}

Let $\mathcal{C}$ be a triangulated category and $K(\mathcal{C}) \rightarrow A$ a finite rank quotient of its Grothendieck group. Consider the subspace of stability conditions on $\mathcal{C}$ whose central charge factors through $A$. Let $\operatorname{Stab}_{0}(\mathcal{C})$ be a component of this subspace. Suppose that $\operatorname{Stab}_{0}(\mathcal{C})$ is full in the sense of [3, Definition 4.2], i.e. that the projection $\operatorname{Stab}_{0}(\mathcal{C}) \rightarrow \operatorname{Hom}(A, \mathbb{C})$ is a local homeomorphism. We prove that such a component is complete in the metric. In fact this follows rather quickly from the results of $[2, \S 7,8]$, which show that $\operatorname{Stab}_{0}(\mathcal{C})$ can be covered by closed balls of fixed radius in the metric, each of which is isometric to a complete metric space 1 However, we give a different proof which has the benefit of providing a useful description of the limiting stability condition.

By assumption stability conditions in $\operatorname{Stab}_{0}(\mathcal{C})$ are locally-finite. We sharpen this slightly, to show that they are locally-length in the sense that given $t \in \mathbb{R}$ we can find $\epsilon>0$ such that the quasi-abelian category $\mathcal{P}_{\sigma}(t-\epsilon, t+\epsilon)$ is a quasi-abelian

\footnotetext{
${ }^{1}$ I would like to thank Arend Bayer for pointing out this quick argument.
} 
length category, or length category for short. By this we mean a quasi-abelian category in which every object $a \neq 0$ has a length $l(a) \in \mathbb{N}$ such that any filtration

$$
\cdots \longmapsto a_{i} \longmapsto a_{i+1} \longmapsto \cdots \longmapsto a
$$

by strict subobjects has length $\leq l(a)$ with equailty for some filtration. (See [6] for more on quasi-abelian categories.) Clearly a length category is both noetherian and artinian, so that a locally-length stability condition is locally-finite. However, the locally-length condition is a priori stronger in that it requires the existence of an upper bound on the length of any filtration, not merely that any filtration have finite length.

Remark 3.1. If $\mathcal{A}$ is a noetherian and artinian abelian category the JordanHölder theorem asserts that any $0 \neq a \in \mathcal{A}$ has a finite composition series with simple factors, and that all such composition series have the same length. So this definition of length category agrees with the usual one in case $\mathcal{A}$ is abelian, and any noetherian and artinian abelian category is a length category. Furthermore, for an abelian length category the length descends to a homomorphism $l: K(\mathcal{A}) \rightarrow \mathbb{Z}$ defined on the Grothendieck group. However, it is not clear that the analogues hold in the quasi-abelian setting.

Lemma 3.2. Suppose $I \subset J$ are nested intervals of length $<1$. Then $\mathcal{P}_{\sigma}(I)$ is a quasi-abelian length category whenever $\mathcal{P}_{\tau}(J)$ is. Moreover the length of a in $\mathcal{P}_{\sigma}(I)$ is bounded above by its length in $\mathcal{P}_{\tau}(J)$.

Proof. A strict monomorphism $\imath: a \longmapsto a^{\prime}$ in $\mathcal{P}_{\sigma}(I)$ fits into a triangle $a \stackrel{\imath}{\longrightarrow} a^{\prime} \longrightarrow$ $a^{\prime \prime} \longrightarrow a[1]$ with $a, a^{\prime}, a^{\prime \prime} \in \mathcal{P}_{\sigma}(I)$ and therefore also in $\mathcal{P}_{\tau}(J)$. Hence $\imath$ is a strict monomorphism in $\mathcal{P}_{\tau}(J)$ too. The result follows.

The next result is a slightly stronger version of [3, Lemma 4.4]; the idea of the proof is the same.

Lemma 3.3. Any $\sigma \in \operatorname{Stab}_{0}(\mathcal{C})$ is locally-length, indeed $\mathcal{P}_{\sigma}(I)$ is a quasi-abelian length category whenever $I$ is a closed interval of length $|I|<1$.

Proof. Suppose $\sigma \in \operatorname{Stab}_{0}(\mathcal{C})$ and that the central charge $Z_{\sigma}$ is rational, i.e. has image in $\mathbb{Q}[i]$. Then the image is a discrete subgroup of $\mathbb{C}$. Assume, without loss of generality, that $I \subset(0,1)$. Fix $0 \neq a \in \mathcal{P}_{\sigma}(I)$. Then

$$
K=\left\{z \in \mathbb{C} \mid \frac{1}{\pi} \arg z \in I \text { and } \operatorname{im} z \leq \operatorname{im} Z_{\sigma}(a)\right\}
$$

is compact, and so contains only finitely many points, say $L$, in the image of $Z_{\sigma}$. Any filtration of $a$ in $\mathcal{P}_{\sigma}(I)$ is mapped under $Z_{\sigma}$ to a sequence in $K$. Since $Z_{\sigma}(b) \neq 0$ for $0 \neq b \in \mathcal{P}_{\sigma}(I)$ the images of the subobjects in the filtration are distinct, and hence the filtration cannot have length greater than $L$.

In the general case, since $\operatorname{Stab}_{0}(\mathcal{C})$ is full we can approximate $\sigma$ by a stability condition $\tau$ with rational central charge and such that $\mathcal{P}_{\sigma}(I) \subset \mathcal{P}_{\tau}(J)$ for some closed interval $J$ of length $<1$. The result follows from Lemma 3.2 .

Suppose that $\sigma_{n}$ is a Cauchy sequence in this component. For ease of reading we write $\mathcal{P}_{n}, Z_{n}, \varphi_{n}, m_{n}$ for $\mathcal{P}_{\sigma_{n}}, Z_{\sigma_{n}}, \varphi_{\sigma_{n}}$ and $m_{\sigma_{n}}$.

Proposition 3.4. Let $0 \neq c \in \mathcal{C}$. The lengths of the Harder-Narasimhan $\sigma_{n}$ filtrations of $c$ are bounded. 
Proof. Fix $0<\epsilon<1 / 8$ and $\theta \in \mathbb{R}$. Choose $M$ such that $d\left(\sigma_{m}, \sigma_{n}\right)<\epsilon$ whenever $m, n \geq M$. In particular, for any $n \geq M$,

$$
\mathcal{P}_{n}(-\infty, \theta+\epsilon) \subset \mathcal{P}_{M}(-\infty, \theta+2 \epsilon) \text { and } \mathcal{P}_{n}(\theta-\epsilon, \infty) \subset \mathcal{P}_{M}(\theta-2 \epsilon, \infty) .
$$

Let $\tau_{n}^{<t}$ be the truncation functor right adjoint to the inclusion of $\mathcal{P}_{n}(-\infty, t)$ in $\mathcal{C}$, and so on. Then

$$
\begin{aligned}
\tau_{n}^{<\theta+\epsilon} \tau_{n}^{>\theta-\epsilon} c & =\tau_{n}^{<\theta+\epsilon} \tau_{n}^{>\theta-\epsilon} \tau_{M}^{>\theta-2 \epsilon} c \\
& =\tau_{n}^{>\theta-\epsilon} \tau_{n}^{<\theta+\epsilon} \tau_{M}^{>\theta-2 \epsilon} c \\
& =\tau_{n}^{>\theta-\epsilon} \tau_{n}^{<\theta+\epsilon} \tau_{M}^{<\theta+2 \epsilon} \tau_{M}^{>\theta-2 \epsilon} c .
\end{aligned}
$$

Hence the number of $\sigma_{n}$-semistable factors of $c$ with phase in $(\theta-\epsilon, \theta+\epsilon)$ is the same as the number of $\sigma_{n}$-semistable factors of $\tau_{M}^{<\theta+2 \epsilon} \tau_{M}^{>\theta-2 \epsilon} c$ with phase in $(\theta-\epsilon, \theta+\epsilon)$. However, the Harder-Narasimhan $\sigma_{n}$-filtration of $\tau_{M}^{<\theta+2 \epsilon} \tau_{M}^{>\theta-2 \epsilon} c$ is contained within

$$
\mathcal{P}_{n}(\theta-3 \epsilon, \theta+3 \epsilon) \subset \mathcal{P}_{M}(\theta-4 \epsilon, \theta+4 \epsilon)
$$

and the latter is a length category by Lemma 3.3 . Therefore there is a uniform (in $n \geq M$ ) bound on the number of $\sigma_{n}$-semistable factors of $c$ with phase in $(\theta-\epsilon, \theta+\epsilon)$. Note that $M$ depends only on $\epsilon$, and not on $\theta$. Therefore, covering $\left[\varphi_{M}^{-}(c)-\epsilon, \varphi_{M}^{+}(c)+\epsilon\right]$ by finitely many intervals of the form $(\theta-\epsilon, \theta+\epsilon)$ we obtain a uniform bound on the length of the Harder-Narasimham $\sigma_{n}$-filtration of $c$ whenever $n \geq M$.

For $\theta \in \mathbb{R}$ we define the limiting semistable objects of phase $\theta$ to be

$$
\mathcal{P}(\theta)=\left\langle 0 \neq c \in \mathcal{C} \mid \varphi_{n}^{ \pm}(c) \rightarrow \theta\right\rangle .
$$

The next lemma shows that limiting semistable objects have non-zero limiting central charge.

Lemma 3.5. Let $0 \neq c \in \mathcal{C}$. If $\varphi_{n}^{+}(c)-\varphi_{n}^{-}(c) \rightarrow 0$ as $n \rightarrow \infty$ then $Z_{n}(c) \not \rightarrow 0$.

Proof. If $\varphi_{n}^{+}(c)-\varphi_{n}^{-}(c) \rightarrow 0$ then $m_{n}(c)-\left|Z_{n}(c)\right| \rightarrow 0$. Therefore $Z_{n}(c) \rightarrow 0$ implies $m_{n}(c) \rightarrow 0$, contradicting the assumption that $\sigma_{n}$ is Cauchy.

Proposition 3.6. Each $0 \neq c \in \mathcal{C}$ has a filtration by limiting semistable objects:

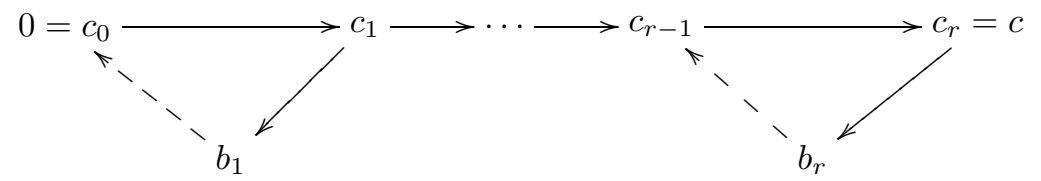

where the factors $b_{i} \in \mathcal{P}\left(\theta_{i}\right)$ with $\theta_{1}>\cdots>\theta_{r}$.

Proof. By Proposition 3.4 there is some $L \in \mathbb{N}$ such that the Harder-Narasimhan $\sigma_{n}$-filtration of $c$ has length $\leq L$ for all sufficiently large $n$. We prove the result by induction on $L$. The case $L=1$ is easy, for in this case $c$ is $\sigma_{n}$-semistable for all sufficiently large $n$ and so $\varphi_{n}^{-}(c)-\varphi_{n}^{+}(c) \rightarrow 0$ (is in fact 0 for sufficiently large $n$ ) and $c$ is itself a limiting semistable. Now assume that the result is true for any object whose Harder-Narasimhan $\sigma_{n}$-filtrations are of length $<L$ for all sufficiently large $n$.

Since $\sigma_{n}$ is a Cauchy sequence both $\varphi_{n}^{+}(c)$ and $\varphi_{n}^{-}(c)$ converge. Suppose that $\varphi_{n}^{+}(c)-\varphi_{n}^{-}(c) \rightarrow \alpha$. If $\alpha=0$ then $c$ is a limiting semistable and the result holds. So we may assume $\alpha>0$. Choose $0<\epsilon<\alpha / 2(L+1)$. Choose $M$ so that $d\left(\sigma_{m}, \sigma_{n}\right)<\epsilon$ 
whenever $m, n \geq M$. For any $n \geq M$ we can find successive semistable factors in the Harder-Narasimhan $\sigma_{n}$-filtration of $c$ whose phases differ by more than

$$
\frac{\alpha-2 \epsilon}{L}>\frac{2(L+1) \epsilon-2 \epsilon}{L}=2 \epsilon \text {. }
$$

Splitting the Harder-Narasimhan $\sigma_{n}$-filtration of $c$ between the above-mentioned factors one obtains a triangle $c^{\prime} \rightarrow c \rightarrow c^{\prime \prime} \rightarrow c^{\prime}[1]$ with $c^{\prime}, c^{\prime \prime} \neq 0$ and such that $\varphi_{n}^{-}\left(c^{\prime}\right)>\varphi_{n}^{+}\left(c^{\prime \prime}\right)$ whenever $n \geq M$. Thus the Harder-Narasimhan $\sigma_{n}$-filtration of $c$ for any $n \geq M$ is obtained by concatenating those of $c^{\prime}$ and $c^{\prime \prime}$. It follows that the Harder-Narasimhan $\sigma_{n}$-filtrations of $c^{\prime}$ and $c^{\prime \prime}$ must have length $<L$ for any $n \geq M$. Hence, by the inductive hypothesis, both $c^{\prime}$ and $c^{\prime \prime}$ have filtrations by limiting semistable objects of decreasing phase. Moreover, the minimal phase of a factor of $c^{\prime}$ is at least as large as the maximal phase of a factor of $c^{\prime \prime}$. The result follows by concatenating these limiting Harder-Narasimhan filtrations of $c^{\prime}$ and $c^{\prime \prime}$.

Theorem 3.7. Let $\mathrm{Stab}_{0}(\mathcal{C})$ be a full component of the space of locally-finite stability conditions on $\mathcal{C}$ whose central charges factor through a given finite rank quotient of the Grothendieck group. Then $\operatorname{Stab}_{0}(\mathcal{C})$ is complete in the natural metric.

Proof. Let $\sigma_{n}$ be a Cauchy sequence. Then the central charges $Z_{n}$ converge, say to $Z: K(\mathcal{C}) \rightarrow \mathbb{C}$. We claim that $\sigma=(Z, \mathcal{P})$ is a locally-length stability condition with $\sigma_{n} \rightarrow \sigma$. It is immediate from the definition of limiting semistable objects that $\mathcal{P}(\theta+1)=\mathcal{P}(\theta)[1]$. By Lemma 3.5] if $0 \neq c \in \mathcal{P}(\theta)$ then $Z(c)=m \exp (i \pi \theta)$ for some $m>0$. If $c \in \mathcal{P}(\theta)$ and $c^{\prime} \in \mathcal{P}\left(\theta^{\prime}\right)$ with $\theta>\theta^{\prime}$ then, for sufficiently large $n$, we have $\varphi_{n}^{-}(c)>\varphi_{n}^{+}\left(c^{\prime}\right)$ so that $\operatorname{Hom}\left(c, c^{\prime}\right)=0$. The existence of Harder-Narasimhan filtrations is guaranteed by Proposition 3.6. Hence $\sigma$ is a stability condition. If the kernel of $K(\mathcal{C}) \rightarrow A$ is annihilated by each of the $Z_{n}$ then it is also annihilated by $Z$, i.e. the central charge of $\sigma$ factors through $A$.

Given $\epsilon>0$ we can choose $M \in \mathbb{N}$ so that $d\left(\sigma_{m}, \sigma_{n}\right)<\epsilon$ for $m, n \geq M$. Then $\mathcal{P}(t-\epsilon, t+\epsilon) \subset \mathcal{P}_{m}(t-2 \epsilon, t+2 \epsilon)$ whenever $m \geq M$. Hence $\sigma$ is locally-length by Lemma 3.2 in particular $\sigma$ is locally-finite. By construction $\sigma_{n} \rightarrow \sigma$, so that $\sigma \in \operatorname{Stab}_{0}(\mathcal{C})$ and the component is complete.

Remark 3.8. The condition that $\operatorname{Stab}_{0}(\mathcal{C})$ is a full component of the space of locally-finite stability conditions on $\mathcal{C}$ whose central charges factor through a given finite rank quotient of the Grothendieck group enters only in the proof that the lengths of the Harder-Narasimhan $\sigma_{n}$-filtrations of a fixed object are bounded as $n \rightarrow \infty$. Hence Theorem 3.7 shows that any Cauchy sequence in the space of locally-finite stability conditions for which this is true converges.

\section{EXAMPLES}

In general the metric $d$, and the induced quotient metric on $\operatorname{Stab}(\mathcal{C}) / \mathbb{C}$, are hard to compute. In this section, under the assumption that the phases of $\sigma$-semistables are dense in $\mathbb{R}$, we compute the restricted metric on the orbit $\sigma G$, where $G$ is the universal cover of $G L_{2}^{+} \mathbb{R}$, and show that it is independent of $\sigma$. In this case the induced metric on $\sigma G / \mathbb{C} \cong \mathbb{H}$ is half the standard hyperbolic metric on the upper half-plane. This allows us to compute the metric on $\operatorname{Stab}(X)$ whenever $X$ is a smooth complex projective curve of genus $\geq 1$, and verify directly that the metrics are complete in these cases. 
Proposition 4.1. Suppose $\sigma \in \operatorname{Stab}(\mathcal{C})$ is a stability condition for which the phases of semistable objects are dense in $\mathbb{R}$. Then the $G$ orbit through $\sigma$ is free, the restriction of the metric d to it is independent of $\sigma$ and thus gives a well-defined metric $d_{G}$ on $G$. This metric is invariant under the action of $G$ by left multiplication and is complete. The induced metric on $\sigma G / \mathbb{C} \cong \mathbb{H}$ is half the hyperbolic metric $d_{\text {hyp }}$ on the upper half-plane.

Proof. Let $g \in G$ correspond to the pair $\left(T_{g}, \theta_{g}\right)$ where $T_{g} \in G L_{2}^{+} \mathbb{R}$ and $\theta_{g}: \mathbb{R} \rightarrow \mathbb{R}$ is increasing with $\theta_{g}(t+1)=\theta_{g}(t)+1$ and induces the same map as $T_{g}$ on the circle $\mathbb{R} / 2 \mathbb{Z}=\mathbb{R}^{2}-\{0\} / \mathbb{R}_{>0}$. Since $\sigma$ and $\sigma g$ have the same semistable objects and Harder-Narasimhan filtrations

$$
\begin{aligned}
d(\sigma g, \sigma) & =\sup _{\text {semistable } c} \max \left\{\left|\varphi_{\sigma g}(c)-\varphi_{\sigma}(c)\right|,\left|\log \frac{m_{\sigma g}(c)}{m_{\sigma}(c)}\right|\right\} \\
& =\sup _{\text {semistable } c} \max \left\{\left|\theta_{g}\left(\varphi_{\sigma}(c)\right)-\varphi_{\sigma}(c)\right|,\left|\log \frac{\left|T_{g} Z_{\sigma}(c)\right|}{\left|Z_{\sigma}(c)\right|}\right|\right\} \\
& =\sup _{\text {semistable } c} \max \left\{\left|\theta_{g}\left(\varphi_{\sigma}(c)\right)-\varphi_{\sigma}(c)\right|,\left|\log \left(\left|T_{g} v\right|\right)\right|\right\}
\end{aligned}
$$

where $v=Z_{\sigma}(c) /\left|Z_{\sigma}(c)\right|$ is the unit vector in the direction of $Z_{\sigma}(c) \in \mathbb{R}^{2}$. Under the assumption that phases of semistables are dense we thus have

$$
\begin{aligned}
d(\sigma g, \sigma) & =\max \left\{\sup _{t}\left|\theta_{g}(t)-t\right|, \sup _{|v|=1}\left|\log \left(\left|T_{g} v\right|\right)\right|\right\} \\
& =\max \left\{\left\|\theta_{g}-\mathrm{id}\right\|, \log \left\|T_{g}\right\|, \log \left\|T_{g}^{-1}\right\|\right\}
\end{aligned}
$$

which depends only on $g$, and not on $\sigma$. Set $\Delta(g)=d(\sigma g, \sigma)$. It is easy to check that $\Delta(g)=0$ if and only if $g=1$, so that

$$
d_{G}(g, h)=d(\sigma g, \sigma h)=d\left((\sigma h) h^{-1} g, \sigma h\right)=\Delta\left(h^{-1} g\right)
$$

is a metric on $G$, and the orbit $\sigma G$ is free. Note that

$$
d_{G}(f g, f h)=\Delta\left((f h)^{-1} f g\right)=\Delta\left(h^{-1} g\right)=d_{G}(g, h)
$$

so that $d_{G}$ is invariant under left multiplication. The explicit description shows that $d_{G}$ is complete.

We have identifications $G / \mathbb{C} \cong G L_{2}^{+} \mathbb{R} / \mathbb{C}^{*} \cong S L_{2} \mathbb{R} / S O(2) \cong \mathbb{H}$ where the last isomorphism is given by

$$
\left(\begin{array}{ll}
a & b \\
c & d
\end{array}\right) \longmapsto \frac{a i+b}{c i+d}
$$

Under this identification the left multiplication action of $S L_{2} \mathbb{R}$ corresponds to its action by Möbius transformations on $\mathbb{H}$. Hence the induced quotient metric on $\sigma G / \mathbb{C} \cong \mathbb{H}$ is invariant under the Möbius action of $S L_{2} \mathbb{R}$. To pin down the metric it therefore suffices to compute the distance between $i$ and $a i$ for real $a \geq 1$. This is given by $\inf _{\lambda \in \mathbb{C}} \Delta(g \lambda)$ where

$$
T_{g}=\left(\begin{array}{cc}
\sqrt{a} & 0 \\
0 & 1 / \sqrt{a}
\end{array}\right) \text { and } \theta_{g}(t)=\frac{1}{\pi} \tan ^{-1}\left(\frac{\tan (\pi t)}{a}\right) .
$$

By direct computation we find that $\inf _{\lambda \in \mathbb{C}} \Delta(g \lambda)=\frac{1}{2} \log a=\frac{1}{2} d_{\text {hyp }}(i, a i)$.

If $X$ is a smooth complex projective curve of genus $\geq 1$ then $\operatorname{Stab}(X) \cong G$ is a single orbit [5]. Furthermore, the phases of semistables are dense since, for the standard stability conditon, there are semistable sheaves of any rational slope. 
(Both of these facts are false for the genus 0 case.) Therefore the spaces of stability conditions are isometric to $\left(G, d_{G}\right)$ for any such $X$, and in particular are complete. Furthermore, $\operatorname{Stab}(X) / \mathbb{C}$ is isometric to the upper half-plane with metric $\frac{1}{2} d_{\text {hyp }}$.

\section{Tilting AND HEARTS OF STABILITY CONDITIONS}

A torsion theory in an abelian category $\mathcal{A}$ is a pair $(\mathcal{T}, \mathcal{F})$ of subcategories such that $\mathcal{F} \subset \mathcal{T}^{\perp}$ and every $a \in \mathcal{A}$ sits in a short exact sequence $0 \rightarrow t \rightarrow a \rightarrow f \rightarrow 0$ with $t \in \mathcal{T}$ and $f \in \mathcal{F}$, see for example [1, Definition 1.1]. In fact $\mathcal{F}=\mathcal{T}^{\perp}$ and $\mathcal{T}={ }^{\perp} \mathcal{F}$ so that it is only necessary to specify either $\mathcal{T}$ or $\mathcal{F}$.

Let $\mathcal{A}$ be the heart of a $t$-structure $\mathcal{D}$. A torsion theory $\mathcal{T}$ in $\mathcal{A}$ determines a new $t$-structure $\langle\mathcal{D}, \mathcal{T}[-1]\rangle$, see [4, Proposition 2.1]: we say this new $t$-structure is obtained from the original by left tilting at $\mathcal{T}$ and denote its heart by $L_{\mathcal{T}} \mathcal{A}$. Explicitly $L_{\mathcal{T}} \mathcal{A}=\langle\mathcal{F}, \mathcal{T}[-1]\rangle$. A torsion theory also determines another $t$-structure $\left\langle\mathcal{D}^{\perp}, \mathcal{F}[1]\right\rangle^{\perp}$ by a 'double dual' construction. This is the shift by [1] of the other: we say it is obtained by right tilting at $\mathcal{T}$ and denote the new heart $\langle\mathcal{F}[1], \mathcal{T}\rangle$ by $R_{\mathcal{T}} \mathcal{A}$. Left and right tilting are inverse to one another: $\mathcal{F}$ is a torsion theory in $L_{\mathcal{T}} \mathcal{A}$ and right tilting with respect to this we recover the original heart $\mathcal{A}$. In the opposite direction, given a $t$-structure $\mathcal{E}$ with $\mathcal{D} \subset \mathcal{E} \subset \mathcal{D}[-1]$ Beligiannis and Reiten [1, Theorem 3.1] show that

$$
\left.\mathcal{T}=\left(\mathcal{E} \cap \mathcal{D}^{\perp}\right)[1]=\langle a \in \mathcal{A}| a=H^{1} e \text { for some } e \in \mathcal{E}\right\rangle
$$

determines a torsion theory in $\mathcal{A}$ such that $L_{\mathcal{T}} \mathcal{A}$ is the heart of $\mathcal{E}$.

Lemma 5.1. If $d(\sigma, \tau)<1 / 2$ then $\mathcal{A}_{\tau}$ can be obtained from $\mathcal{A}_{\sigma}$ by performing a left and then a right tilt. (Of course one or both of these tilts may be trivial.)

Proof. Recall that $\mathcal{A}_{\sigma}=\mathcal{P}_{\sigma}(0,1]$ is the heart of the $t$-structure $\mathcal{P}_{\sigma}(0, \infty)$, and analogously for $\mathcal{A}_{\tau}$. Since $d(\sigma, \tau)<1 / 2$ there are inclusions of $t$-structures

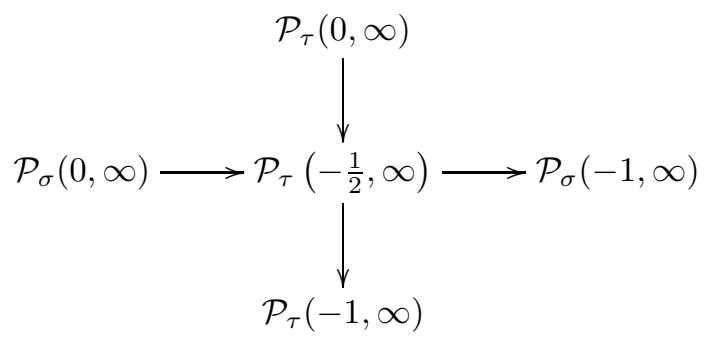

So $\mathcal{P}_{\tau}(-1 / 2, \infty)$ determines torsion theories $\mathcal{T}$ in $\mathcal{A}_{\sigma}$ and $\mathcal{T}^{\prime}$ in $\mathcal{A}_{\tau}$ with

$$
L_{\mathcal{T}} \mathcal{A}_{\sigma}=\mathcal{P}_{\tau}(-1 / 2,1 / 2]=L_{\mathcal{T}}, \mathcal{A}_{\tau} .
$$

Hence $\mathcal{A}_{\tau}=R_{\mathcal{F}^{\prime}} L_{\mathcal{T}} \mathcal{A}_{\sigma}$.

Corollary 5.2. If $\sigma$ and $\tau$ are in the same component of $\operatorname{Stab}(\mathcal{C})$ then the hearts $\mathcal{A}_{\sigma}$ and $\mathcal{A}_{\tau}$ are related by a finite sequence of left and right tilts.

Proof. The connected components of $\operatorname{Stab}(\mathcal{C})$ are the path components. Choose a path from $\sigma$ to $\tau$, cover it with finitely many balls of diameter $<1 / 2$ and apply Lemma 5.1

For a heart $\mathcal{A}$ let $U(\mathcal{A})=\left\{\sigma \in \operatorname{Stab}(\mathcal{C}) \mid \mathcal{A}_{\sigma}=\mathcal{A}\right\}$. Note that $U(\mathcal{A})$ may be empty and need not be either open or closed. The next result describes the hearts of stability conditions in the closure of $U(\mathcal{A})$. 
Corollary 5.3. Suppose $\sigma_{n}$ is a sequence in $U(\mathcal{A})$ with $\sigma_{n} \rightarrow \sigma$. Then $\mathcal{A}_{\sigma}=R_{\mathcal{T}} \mathcal{A}$ for the torsion theory with

$$
\mathcal{T}=\left\langle 0 \neq a \in \mathcal{A} \mid \varphi_{n}^{-}(a) \not \rightarrow 0\right\rangle
$$

and $\mathcal{F}=\left\langle 0 \neq a \in \mathcal{A} \mid \varphi_{n}^{+}(a) \rightarrow 0\right\rangle$.

Proof. It is easy to check that $\mathcal{T}$ is a torsion theory; the required short exact sequences arise from the description of the Harder-Narasimhan $\sigma$-filtration as a limiting filtration. The description of the limiting semistable objects shows that $R_{\mathcal{T}} \mathcal{A} \subset \mathcal{A}_{\sigma}$. Hence they are equal as hearts of distinct non-degenerate $t$-structures cannot be nested.

It is not always the case that a torsion theory has the form $\langle 0 \neq a \in \mathcal{A}| \varphi_{n}^{-}(a) \not \rightarrow$ $0\rangle$ for some Cauchy sequence $\sigma_{n}$ of stability conditions, and it is not true that $\mathcal{B}=R_{\mathcal{T}} \mathcal{A}$ implies $\overline{U(\mathcal{A})} \cap U(\mathcal{B}) \neq \emptyset$. For example, let $\mathcal{A}$ be the category of representations of the Kronecker quiver. This is well-known to be derived equivalent to the coherent derived category of $\mathbb{P}^{1}$ and (after fixing an appropriate equivalence) $\mathcal{A}=L_{\mathcal{T}} \operatorname{Coh}\left(\mathbb{P}^{1}\right)$ where $\mathcal{T}={ }^{\perp}\langle\mathcal{O}(d) \mid d<0\rangle$. However there is no sequence $\sigma_{n}$ in $U(\mathcal{A})$ with $\sigma_{n} \rightarrow \sigma$ where $\mathcal{A}_{\sigma}=\operatorname{Coh}\left(\mathbb{P}^{1}\right)$; see [7, §3.2] for details.

\section{REFERENCES}

[1] A. Beligiannis and I. Reiten. Homological and homotopical aspects of torsion theories. Mem. Amer. Math. Soc., 188(883):viii+207, 2007.

[2] T. Bridgeland. Stability conditions on triangulated categories. Ann. of Math. (2), 166(2):317$345,2007$.

[3] T. Bridgeland. Stability conditions on K3 surfaces. Duke Math. J., 141(2):241-291, 2008.

[4] D. Happel, I. Reiten, and S. Smalø. Tilting in abelian categories and quasitilted algebras. Mem. Amer. Math. Soc., 120(575), 1996.

[5] E. Macrì. Stability conditions on curves. Math. Res. Lett., 14(4):657-672, 2007.

[6] J.-P. Schneiders. Quasi-abelian categories and sheaves. Mém. Soc. Math. Fr. (N.S.), (76):vi+134, 1999.

[7] J. Woolf. Stability conditions, torsion theories and tilting. J. London Math. Soc., 2010. 